\title{
COVID-19 infection in a HIV positive health care worker: first case report from a tertiary care hospital of North India
}

\author{
Ritin Mohindra ${ }^{1} \cdot$ Poonam Kanta $^{2} \cdot$ Pradakshana Porchezhian $^{1} \cdot$ Kapil Goyal $^{2}$ •

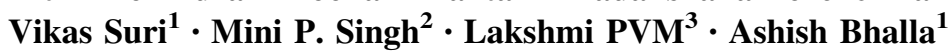

Received: 24 July 2020/Accepted: 18 January 2021/Published online: 7 April 2021

(C) Indian Virological Society 2021

\begin{abstract}
People living with Human Immunodeficiency Virus (PLHIV) are at greater risk of developing prolonged illness due to COVID 19 leading to longer duration of virus shedding owing to their underlying immune defects. The present study compared SARS-CoV-2 infection developing at the same time among two health care workers living with and without a history of HIV and working in the same ward of a tertiary care hospital of North India. A higher viral load was reported in the SARS-CoV-2 infected worker who was immunocompromised as compared to immunocompetent patient $(19,193$ copies $/ \mu \mathrm{L}$ vs 9.4 copies $/ \mu \mathrm{L})$. In this preliminary case report, no difference was observed in the clinical presentation of both patients at the time of diagnosis. Further studies are required to investigate the COVID-19 susceptibility and severity among HIV-infected patients.
\end{abstract}

Keyword COVID-19 and human immunodeficiency virus - people living with human immunodeficiency virus . immunocompromised

Immunocompromised individuals are at a high risk of acquiring opportunistic infections. SARS CoV-2 has also been reported among immunocompromised individuals

Ritin Mohindra and Poonam Kanta have contributed equally to this work.

Mini P. Singh

minipsingh@gmail.com

1 Department of Internal Medicine, PGIMER, Chandigarh, India

2 Department of Virology, PGIMER, Chandigarh, India

3 School of Public Health, PGIMER, Chandigarh, India with prolonged shedding. Most of the clinical manifestations mainly depend upon host factors and are associated with inflammatory response associated with COVID-19. However, with the availability of antiretroviral treatment and better diagnostic facilities, the prevalence of HIV has been declining since 2007 and reported as $0.22 \%$ in the year 2017 (http://naco.gov.in/sites/default/files/HIV\% 20Estimations\%202017\%20Report_1.pdf). However, there is limited literature available regarding COVID-19 among PLHIV [8].

The SARS-CoV-2 infection usually starts with mild flulike symptoms leading to pneumonia in the later stages in some patients. A lot of heterogeneity in the symptoms is being observed in the populations affected with COVID19 , a large proportion of whom are mostly asymptomatic but almost 6-8\% may require hospitalization (https:// cddep.org/covid-19/modeling-the-21-day-indian-lockdown/). The hospitalization rates are lowest in $0-4$ years of age $(0.3 \%)$ and highest in patients $>65$ years $(13.8 \%$ ) (https:// www.cdc.gov/mmwr/volumes/69/wr/mm6915e3.htm). It has been seen that the majority of the COVID-19 patients with comorbidities such as hypertension, diabetes, cardiovascular disease, chronic lung disease, chronic kidney disease etc. are likely to be symptomatic.

It is well known that immunocompromised individuals are at a higher risk of acquiring viral infections and also these infections tend to persist for a longer duration of time in such individuals [4]. People living with Human Immunodeficiency Virus (PLHIV) represent a unique cohort due to their underlying immune defects. Human immunodeficiency virus (HIV) is known to invade the immune system thereby decreasing the $\mathrm{CD} 4^{+} \mathrm{T}$ cells count [2]. A special attention is required for PLHIV with low CD4 cell count, high viral load, advanced stage of HIV 
disease and lastly those who are not taking effective ART treatment.

At present, there is scarcity of literature pertaining to the natural course of SARS CoV-2 among PLHIV. However, in a recently published case series $(n=5)$, it was seen that at the time of hospitalization HIV infected patients had mild $(40 \%)$, moderate $(20 \%)$ or severe symptoms $(40 \%)$. All the patients were on antiretroviral therapy (ART) during hospitalization and 4 out of $5(80 \%)$ recovered and were discharged from the hospital [3].

In the present case report, authors describe a comparison of SARS CoV-2 viral load and duration of shedding among a immunocompromised health care worker (PLHIV) (Patient 1) and an immunocompetent (Non-PLHIV) health care worker (Patient 2); both of them were working in COVID-19 areas (Isolation wards) of a tertiary care hospital of north India at the same time and tested positive for COVID-19 on the same day. These cases were diagnosed during routine screening of healthcare workers (HCW's), wherein a total of $43 \mathrm{HCW}$ 's who were involved in caring for COVID-19 patients were screened and tested for COVID-19. The demographic, clinical and laboratory details of both HCWs are mentioned in Table 1. Both the above mentioned HCWs were asymptomatic and were enrolled in the institute trial on hydroxychloroquine (HCQ) prophylaxis. However, patient 1 was not started on HCQ prophylaxis whereas patient 2 had received a loading dose of HCQ prophylaxis $(800 \mathrm{mg}$ ). The HCW's did not report any breach in personal protective equipment (PPE) during patient handling. Patient 1 had $\mathrm{CD} 4^{+}$count 726 cells per $\mu \mathrm{L}$ at the time of admission but also suffered from ulcerative colitis for which he was taking Mesalamine (1.2 gm once a day) at the time of admission. Clinical and physical examination were unremarkable in both the patients at the time of diagnosis. During hospitalization, both of them received therapeutic hydroxychloroquine but no specific antiviral therapy was given in either patient. Patient 1 was not taking any antiretroviral treatment for HIV. The only risk factor which could be attributed to acquisition of infection was history of recent contact with a positive patient within the last 14 days.

The viral load in the immunocompromised patient was found to be higher as compared to immunocompetent patient $(19,193$ copies/ $\mu \mathrm{L}$ and 9.4 copies/ $\mu \mathrm{L}$ respectively) at the time of diagnosis despite having the same period of contact with the laboratory confirmed case of COVID-19. None of the contacts of the latter were found to be infected with COVID-19. However, all the household contacts of immunocompromised patient were found to be positive for SARS-CoV2 RNA. Additionally, it was seen that time to achieve negativity for SARS CoV-2 by Real-time PCR was also more in Patient 1 (14 days) as compared to Patient 2 (7 days). However, on Day 14, both the patients tested negative ( 2 consecutive samples, tested $24 \mathrm{~h}$ apart) and patient 2 was discharged to home (two negative consecutive reports) as per national guidelines.

Even though the COVID-19 pandemic has rapidly spread worldwide, little is known about its impact and outcomes in immunocompromised patients. The severity and clinical outcome of many viral infections such as Respiratory syncytial virus (RSV) and influenza (seasonal and pandemic H1N1) in PLHIV patients has been described in immunocompromised and immunocompetent cases and the presentation varies from full recovery to death [6]. These studies have concluded that comorbid conditions might be responsible for the increased hospitalization and mortality in immunocompromised (PLHIV) with lower $\mathrm{CD}^{+}$count [11]. Adults of any age with underlying medical conditions such as cancer, asthma, diabetes, obesity, heart diseases and immunocompromised state (weak immune system or organ transplant patients) are at higher risk for developing severe illness and hospitalization than others (https://www.cdc.gov/coronavirus/2019-ncov/coviddata/investigations-discovery/assessing-risk-factors.html).

The reported positivity rate of COVID-19 in PLHIV is about $0.68 \%$ as shown in a recent study from China which was similar to the infection rate in the normal population [5]. Likewise, during the SARS and MERS outbreaks, the infection rate in PLHIV was reported to be low (https:// www.who.int/news-room/q-a-detail/q-a-on-covid-19-hivand-antiretrovirals). Overall, the prevalence of PLHIV in India is about $0.2 \%$ and co-infection with COVID-19 has been infrequently reported from India (https://www.avert. org/professionals/hiv-around-world/asia-pacific/india). The HIV infection has not been reported to increase the severity of the COVID-19 infection. This was the first case reported in a tertiary care hospital of north India wherein a health care worker who was PLHIV was infected with COVID-19 and his clinical course was compared with non-PLHIV patient who was diagnosed to be SARS CoV-2 positive at the same time during screening. Because of the difference in the viral loads, the immunocompromised patient (PLHIV) had a longer time to swab negativity as compared to the other patient. The PLHIV patients with their low $\mathrm{CD}^{+}{ }^{+}$counts and not on any retroviral treatment are at a higher risk of other infections (bacterial or fungal) which may further worsen the condition of COVID-19 infected patients as has been recently reported in Gujarat, India (https://timesofindia.indiatimes.com/city/ahmedabad/hiv-veman-beats-covid).

It is difficult to explain the source of acquisition of SARS CoV-2 infection in both of the HCWs as both were regularly working in the isolation ward of the hospital and taking adequate protective measures while handling the COVID-19 patients. The possibility that both the HCWs 
Table 1 Laboratory profile of patients

Immunocompromised patient $(\mathrm{HIV}+)$

Immunocompetent patient (HIV-)

Demographics

Age

30

$\mathrm{M}$

yes

Health care worker

Hospital

\section{Comorbidities}

HIV diagnosis (since)

HIV viral load on admission

ART-regimen on admission

Other diseases

Clinical findings on admission

Contact with COVID-19 positive patient

Duration of symptoms, days

Diagnosis, symptoms

Body temperature

Blood pressure $(\mathrm{mm} \mathrm{Hg})$

Respiratory rate (breaths per min)

Heart rate (beats per min)

Chest x-ray

Requirement of ventilation

\section{Laboratory findings}

Haemoglobin $(\mathrm{g} / \mathrm{dl})$

WBC (cells per microliter)

Neutrophils (\%)

Lymphocyte (\%)

Eosinophils (\%)

Monocytes (\%)

Platelet (cell per microliter)

C-reactive protein $(\mathrm{mg} / \mathrm{dL})$

Procalcitonin $(\mathrm{ng} / \mathrm{mL})$

Ferritin (ng/mL)

CD4 (cell per microliter)

Treatment at the time admission

ART +

Other antiviral

Antibiotics

Admitted to ICU

Length of hospital stay

Other treatment

Hydroxychloroquine as prophylaxis

Outcome

Family history

Family members affected with HIV

HIV treatment if any

Family members affected with COVID

Symptoms appeared in Days

Treatment for COVID-19

Days of contact with index case

\section{PGIMER}

2019

ND

No treatment

Ulcerative colitis

Yes

0

Asymptomatic

Afebrile

$138 / 80$

18

88

Normal

No

12.4

7600

40

51

1.5

6

81,000

1.02

0.02

37.6

726

No

No

No

ward

14

Mesalamine (1.2gm OD)

No

Cured

NO

NO

Yes

Asymptomatic

Not given

On Daily basis
55

M

Yes

PGIMER

NO

ND

NO

NO

Yes

0

Asymptomatic

Afebrile

132/92

18

67

Normal

No

11.5

3500

57

24

8

10

75,000

0.02

0.02

112.8

ND

No

No

No

14

Not given

Yes

Cured

NO

NO

NO

Asymptomatic

Not given

On daily basis 
Table 1 continued

\begin{tabular}{lll}
\hline & Immunocompromised patient (HIV+) & Immunocompetent patient (HIV-) \\
\hline $\begin{array}{l}\text { Comorbidities } \\
\text { COVID-19 Testing }(N \text {-gene })\end{array}$ & No & No \\
Patient & 19,193 copies per microliter & 9.4 copies per microliter \\
Repeat sampling & & \\
7th days & 194.85 copies per microliter & Negative \\
14th days & Negative & Negative \\
\hline
\end{tabular}

might have acquired the infection through community cannot be entirely ruled out.

In a recent a case series of 33 PLHIV cases infected with COVID-19, a mortality of $9 \%$ has been reported and recovery has been reported among $91 \%$ of the patients. The clinical course and risk of acquiring COVID-19 in PLHIV patients on effective ART treatment has been reported to be the same as in the general population [7]. The patients with low CD count, no effective ART treatment, underlying medical conditions and old age group might develop severe symptoms due to weak immune system and high inflammatory response (https://www.hiv.gov/hiv-basics/stayingin-hiv-care/other-related-health-issues/coronavirus-covid19) $[10]$.

The treatment of patients diagnosed with COVID-19 should be individualized and depends on the severity of illness and presence of other comorbidities. Most of the asymptomatic and mild cases recover spontaneously. However, antivirals, steroids and immunomodulation therapy have been found to be useful among moderate and severe cases. An uninterrupted availability of ART to PLHIV patients along with refills for longer duration may reduce the contact with health facilities leading to a reduced risk of COVID-19 [7, 9].

In the present case report both HCWs were asymptomatic but PLHIV presenting with respiratory symptoms should also be investigated for other opportunistic infections which are commonly prevalent [3]. Asymptomatic immunocompromised individuals may also act as super spreaders especially in health care set up due to prolonged shedding of virus as shown in a recently published case report [1]. Cases of SARS CoV-2 infection among HIV positive patients have been reported from Lucknow and also from Gujarat and both these cases recovered successfully (https://www.hindustantimes.com/cities/man-liv ing-with-hiv-beats-covid-19-in-six-days/story-e9Dc4Lper MiiKTzVC37onN.html).

According to GOI guidelines, due to lack of information regarding effective antiviral treatment for SARS-CoV-2 infection and approved vaccine, only symptom-based treatment is recommended. In severe symptomatic cases reporting with respiratory distress, hypoxia and shock: oxygen therapy needs to be started immediately (https:// www.mohfw.gov.in/pdf/RevisedNationalClinicalManage mentGuidelineforCOVID1931032020.pdf). In severe cases ( $\mathrm{SpO} 2<90 \%$ on room air), mechanical ventilation, corticosteroids and anticoagulants are recommended(https:// www.mohfw.gov.in/pdf/UpdatedClinicalManagementPro tocolforCOVID19dated03072020.pdf). Role of various antivirals for the treatment of SARS CoV-2 is still debatable and HIV specialists must be consulted in this scenario while treating coinfection with SARS-CoV-2 virus because of lack of information on drug-drug interactions (https:// www.covid19treatmentguidelines.nih.gov/special-popula tions/hiv/).

Till date, a safe and effective vaccine has not yet been approved for COVID-19 but several potential candidate vaccines are in different phases of clinical trials. Currently mRNA based COVID-19 vaccine BNT162b2 (developed by Pfizer and BioNTech SE) (https://www.pfizer.com/ news/press-release/press-release-detail/pfizer-and-bion tech-conclude-phase-3-study-covid-19-vaccine) and mRN A-1273 vaccine (developed by Moderna Inc) showed $>$ 94\% efficacy in phase III clinical trials (https://edition.cnn. com/2020/11/16/health/moderna-vaccine-results-corona virus/index.html_ but in Indian scenario the cost, storage of vaccine at particular temperature and massive scale production of vaccine is a mammoth task. India is also carrying out clinical trails of indigenously manufactured inactivated COVID-19 vaccine COVAXIN (developed by Bharat Biotech in collaboration with ICMR). Other candidate COVID-19 vaccines such as Covishield (Phase II), ZyCoV-D (Phase II), SPUTNIK (Phase II) have shown promising results in clinical trials and could be available for public use (https://vaccine.icmr.org.in/covid-19-vac cine). It has been announced that COVID-19 vaccine would be administered to frontline healthcare workers and $>50$ years old individuals with underlying comorbid 
conditions. (https://www.oneindia.com/india/who-willget-covid-19-vaccine-first-in-india-3178041.html). For streamlining the vaccine coverage Intelligence network for vaccination has been developed by GoI (https://www.in. undp.org/content/india/en/home/projects/gavi1.html).

Until an effective vaccine for COVID-19 is being released, HCWs living with HIV need to adhere to donning and doffing protocols of personal protective equipment (PPE) stringently while working in a high-risk area. Appropriate preventive guidelines can be formulated for immunocompromised individuals who are working in a high-risk area. Apart from these general preventive measures such as frequent hand washing, universal masking and appropriate social distancing needs to be reinforced.

Limitations: In the present study, shedding of SARSCoV-2 among different samples such as saliva and stool was not included and moreover a single PLHIV case was discussed and compared with Non-HIV COVID positive patient.

\section{Informed consent}

Institute Ethics committee had approved the retrospective study with waive off for consent of the patient (NK/6445/ Study/685).

Acknowledgement We would like to acknowledge patients involved in the study for their support.

\section{Compliance with Ethical Standards}

Informed Consent Institute Ethics committee had approved the retrospective study with waive off for consent of the patient (NK/ 6445/Study/685).

\section{References}

1. Avanzato VA, Matson MJ, Seifert SN, Pryce R, Williamson BN, Anzick SL, et al. Case study: prolonged infectious SARS-CoV-2 shedding from an asymptomatic immunocompromised cancer patient. Cell. 2020. https://doi.org/10.2139/ssrn.3699798.

2. Beck JM. The immunocompromised host: HIV infection. Proc Am Thorac Soc. 2005;2:423-7.

3. Blanco JL, Ambrosioni J, Garcia F, Martínez E, Soriano A, Mallolas J, et al. COVID-19 in patients with HIV: clinical case series. Lancet HIV. 2020;7:e314-6.

4. Englund J, Feuchtinger T, Ljungman P. Viral infections in immunocompromised patients. Biol Blood Marrow Transpl. 2011;17:S2-5.

5. Guo W, Ming F, Dong Y, Zhang Q, Zhang X, Mo P et al. A survey for COVID-19 among HIV/AIDS patients in two Districts of Wuhan, China. AIDS Patients in Two Districts of Wuhan, China (3/4/2020). 2020.

6. Gupta A, Mody P, Gupta S. A case of respiratory syncytial virus infection in an HIV-positive adult. Case Rep Infect Dis. 2012. https://doi.org/10.1155/2012/267028.

7. Haerter G, Spinner CD, Roider J, Bickel M, Krznaric I, Grunwald $\mathrm{S}$, et al. COVID-19 in people living with human immunodeficiency virus: a case series of 33 patients. Infection. 2020;48:681-6.

8. Huang L, Crothers K. HIV-associated opportunistic pneumonias. Respirology. 2009;14:474-85.

9. Mirzaei H, McFarland W, Karamouzian M, Sharifi H. COVID-19 among people living with HIV: a systematic review. AIDS Behav. 2020. https://doi.org/10.1007/s10461-020-02983-2.

10. Prabhu S, Poongulali S, Kumarasamy N. Impact of COVID-19 on people with HIV: a review. J Virus Eradic. 2020;6:100019.

11. Sheth AN, Althoff KN, Brooks JT. Influenza susceptibility, severity, and shedding in HIV-infected adults: a review of the literature. Clin Infect Dis. 2011;52:219-27.

Publisher's Note Springer Nature remains neutral with regard to jurisdictional claims in published maps and institutional affiliations. 